\title{
PERSPECTIVA SOCIO-PSICOLÓGICA DEL MODELO DE ACEPTACIÓN DE LA TECNOLOGÍA Revisión crítica del trabajo de Torres, Robles y Molina (2011)
}

\section{A SOCIO-PSYCHOLOGICAL PERSPECTIVE OF THE TECHNOLOGY ACCEPTANCE MODEL \\ A critical review of Torres, Robles and Molina's work}

Jesús Manuel LóPez-Bonilla lopezbon@us.es

Universidad de Sevilla. España

Luis Miguel López-Bonilla luismi@us.es

Universidad de Sevilla. España

\section{RESUMEN}

Este trabajo es una revisión crítica del artículo de Torres, Robles y Molina (2011), que ha sido publicado en la Revista Internacional de Sociología (RIS, vol. 69, $n^{\circ} 2$ ). Dicho artículo es de gran interés, pero observamos que hay varios sesgos de los investigadores que restan confianza a los resultados obtenidos.

\section{Palabras claves}

Internet; TAM; Tecnología Información y comunicación.

\section{Abstract}

This paper is a critical review about the article of Torres, Robles and Molina (2011), which has been published in Revista Internacional de Sociología (RIS, vol. $69, n^{0} 2$ ). This article is very interesting, but we observe that there are several biases of the researchers remaining confidence in the results obtained.

\section{KEYWORDS}

Information and Communication Technologies; Internet; TAM. 


\section{INTRODUCCIÓN}

El presente trabajo se plantea como una revisión crítica del artículo Torres, Robles y Molina (2011) que se ha publicado en la Revista Internacional de Sociología. Dicho artículo se basa en un estudio sobre la aceptación de una tecnología en una muestra representativa de españoles, aplicando para ello uno de los modelos más extendidos en la literatura, como es el Modelo de Aceptación de la Tecnología (TAM, Technology Acceptance Model). De este modo, los autores mencionados proponen la hipótesis de potenciar las variables sociodemográficas en el TAM, tratando de ofrecer una versión sociológica del modelo que favorezca al aumento de su poder explicativo.

Torres, Robles y Molina (2011) establecen tres interrogantes en su estudio, como son: (1) ¿cuáles son las variables sociales más influyentes en el uso de Internet?; (2) ¿las variables psicológicas son más influyentes que las variables sociales en el uso de Internet?; y (3) ¿cuál es el papel conjunto de las variables sociales y psicológicas en la explicación del uso de Internet?

Las respuestas dadas a tales preguntas se pueden sintetizar de la siguiente manera: (1) las variables sociales más destacadas son la edad, seguida de la educación; (2) la influencia de las variables sociales es sensiblemente superior a la influencia de las variables psicológicas; y (3) es preciso afrontar un giro sociológico del TAM, es decir, los autores concluyen que la formulación del TAM debe considerar las variables sociales que definen al individuo para favorecer la capacidad explicativa de dicho modelo, indicando la necesidad de construir un nuevo modelo alternativo "más orientado a una línea de explicación netamente sociológica".

En el presente trabajo hacemos referencia al trabajo de Torres, Robles y Molina (2011) porque nos resulta eminentemente interesante y enriquecedor, a la vez que estimulante para replicarlo con una revisión crítica que pretende aportar un mayor conocimiento a la materia, en la medida de nuestras posibilidades. Desde una perspectiva interdisciplinar, estamos de acuerdo con los autores citados en la necesidad de una mayor participación de los factores sociales en el Modelo de Aceptación de la Tecnología. En este sentido, Legris et al. (2003) indican que el TAM es un modelo útil pero necesita incluir variables relacionadas con los procesos de cambios sociales y humanos. Sin embargo, disentimos especialmente en la preponderancia de estos factores respecto a las variables psicológicas. Pero no porque pensemos que los factores psicológicos puedan ser mejores y más precisos, sino porque creemos que hay algunos aspectos básicos en el planteamiento del estudio que nos hacen mantener una gran cautela en los resultados obtenidos, que confirman el hecho de una mayor superioridad de los factores sociales del individuo frente a los psicológicos para explicar el uso de Internet. 


\section{REVISIÓN CRÍTICA}

La revisión que hemos realizado se centra en el contenido teórico y, sobre todo, en el contenido analítico del trabajo de Torres, Robles y Molina (2011). Pero también hemos pretendido aportar nuestra propuesta para estudios posteriores en este ámbito de estudio, en el que estamos familiarizados.

A nivel teórico tan sólo hacemos un apunte respecto a la revisión de la literatura realizada por Torres, Robles y Molina (2011:376). Estos autores describen el Modelo de Aceptación de la Tecnología (TAM) subrayando que "la intención de un agente individual de utilizar una determinada tecnología depende, fundamentalmente, de la actitud de este sujeto hacia la herramienta tecnológica". A este respecto, habría que precisar que el TAM ha evolucionado hacia un modelo que excluye las actitudes. Así, los estudios de Davis, Bagozzi y Warshaw (1992) y Venkatesh y Davis (2000) ponen de manifiesto que las medidas de ajuste de las actitudes hacia el uso de la tecnología no se pueden considerar como suficientes para mantener dicho concepto dentro del modelo, por lo que proponen eliminarlo, quedando la utilidad percibida y la facilidad de uso percibida como los antecedentes directos de las intenciones de uso. Por lo tanto, debemos saber que el TAM2, propuesto por Venkatesh y Davis (2000), ya excluye las actitudes del modelo, incorporando otros factores determinantes, que se pueden dividir en dos grupos, como son los factores relacionados con los procesos de influencia social (normas subjetivas, voluntariedad, imagen y experiencia) y los factores relativos a los procesos cognitivos (relevancia del trabajo, calidad del resultado y demostrabilidad del resultado).

No obstante, López y López (2006) realizan una comparación de las dos versiones del TAM basadas en la inclusión o exclusión de las actitudes (que denominan como TAM Primitivo y TAM, respectivamente), obteniendo como resultado que el primero de ellos, es decir, el TAM original en el que se incluyen las actitudes, proporciona un mejor ajuste, explicando en mayor grado la intención de uso de la tecnología.

Por otro lado, a nivel analítico, apreciamos una serie de sesgos en el estudio de Torres, Robles y Molina (2011) que nos incitan a expresar una elevada cautela en los resultados que han obtenido. Tan sólo para facilitar la explicación de estos sesgos, hemos tratado de agruparlos en tres tipos, como son: (1) sesgo estructural, en relación con la elaboración del propio modelo; (2) sesgo instrumental, referido a la técnica de análisis utilizada; y (3) sesgo metodológico, en cuanto a la operatividad de las variables y los conceptos tratados. Seguidamente, pasamos a detallarlos.

A nuestro juicio, el sesgo estructural se produce en el momento de construir el modelo a partir de la influencia directa de las variables sociales y psicológicas sobre el uso de Internet. EI TAM se elabora en función de, al menos, la relación entre las creencias y las intenciones de uso, que determinan el uso real de la tecnología. Como hemos comentado anteriormente, el TAM2 ha eliminado las actitudes y ha añadido una serie de variables externas, por lo que el proceso que explicara el uso de la tecnología podría incluir también estas otras fases. La relación directa que proponen Torres, Robles 
y Molina (2011) es perfectamente válida, pero creemos que, si pretendemos realizar una comparación con el TAM o el TAM2, habría que tener presente su formulación y plantear los efectos sociales y psicológicos sobre la intención de uso de la tecnología, más que directamente sobre su uso. Como apuntan Venkatesh et al. (2003), el rol de la intención como predictor del comportamiento es crítico y ha sido claramente establecido en la literatura en el ámbito de diferentes disciplinas.

El sesgo instrumental radica en la aplicación del análisis logístico como técnica estadística clásica. Este tipo de análisis se limita a examinar únicamente una relación al mismo tiempo, entre una variable dependiente y un conjunto de variables independientes. Es decir, no evalúan diversas relaciones concatenadas en las que una misma variable es causa de unas variables y consecuencia de otras. Para ello, como indican Hair et al. (2004), es más apropiado y exhaustivo el uso del modelo de ecuaciones estructurales cuando se estudia simultáneamente una serie de relaciones de dependencia y, además, tienen la habilidad de incorporar variables latentes al análisis. EI TAM requiere de esta clase de análisis simultáneo de relaciones e incluye variables latentes, como son los factores psicológicos. No cabe duda de que este modelo implica un conjunto de relaciones complejas que hay que abordar con técnicas que proporcionen una explicación rica. De hecho, en la gran mayoría de estudios sobre el TAM se emplean estas técnicas multivariantes.

En cuanto al sesgo metodológico, hemos distinguido dos aspectos concretos. Por un lado, las escalas de medida de las variables psicológicas y, por otro, la medición del uso de Internet. En el primer caso, las escalas de medida de la utilidad percibida y de la facilidad de uso percibida, que se han tratado en Torres, Robles y Molina (2011), se basan en un tipo de escala numérica con un rango entre 1 (mínima percepción por parte del individuo) y 5 (máxima percepción). A primera vista parece que las dos variables psicológicas son medidas con un único ítem, aunque no se indica nada al respecto. La literatura establece ampliamente que este tipo de escala está basado en varios ítems, al tratar de medir una variable latente. Por ello, en general, un solo ítem no suele ser apropiado para medir cualquier variable psicológica, o bien, en definitiva, cualquier concepto (Hair et al., 2004). En este sentido, todos los estudios sobre el TAM incluyen varios indicadores de cada constructo con objeto de alcanzar una respuesta más precisa. Además, en el caso de utilizar varios ítems, habría que comprobar sus niveles de fiabilidad y validez para el estudio en cuestión.

Respecto a la medición del uso de Internet en el estudio citado está basada en el hecho de haberse conectado en los últimos tres meses a la red. Internet ya es un medio de comunicación de masas que está alcanzando los niveles de difusión de otros medios como la televisión y la radio. Sería inadecuado tratar de identificar a los televidentes o radioyentes preguntándoles si han visto u oído la televisión o la radio alguna vez en los últimos meses. Ocurre lo mismo con los internautas, que no se entiende que lo sea quien se conecta a la red, por ejemplo, una o dos veces al trimestre. Es decir, es necesario hacer más evidente el uso de Internet. Sobre todo, teniendo en cuenta que los datos de la encuesta obtenidos por Torres, Robles y Molina 
(2011) son muy actuales (noviembre de 2008). Por ello, se debe conocer con mayor precisión si un individuo usa Internet y en qué grado lo usa. Se puede preguntar por aspectos objetivos (Teo, Lim y Lai 1999; Roberts y Henderson, 2000; Al-Gahtani 2001) o subjetivos (Agarwal y Prasad, 1997; Porter y Donthu, 2006) sobre el uso de Internet. Así, por ejemplo, Teo, Lim y Lai (1999) utilizan una escala con tres ítems con los que tratan de conocer la frecuencia de uso de Internet, el uso diario de Internet en número de horas y la diversidad de usos de Internet. En cambio, Porter y Donthu (2006) establecen una escala tipo Likert que se compone también de tres indicadores, como son: 1) utilizo Internet bastante a menudo para uso personal, 2) Consumo mucho tiempo en Internet para uso personal, y 3 ) he estado utilizando Internet para uso personal desde hace tiempo.

\section{Conclusiones}

El estudio de Torres, Robles y Molina (2011:376) es muy interesante y sugerente para futuras investigaciones, pero sus resultados deben ser considerados con una gran prudencia. Este hecho está avalado por la comprobación de una serie de sesgos operativos cometidos por los investigadores, que no debemos identificarlos con errores del estudio en ningún caso, sino con amenazas que generan una mayor desconfianza en los resultados obtenidos. Por ello, nos inclinamos por descartar la posibilidad de confirmar la supremacía de las variables sociológicos respecto a las variables psicológicas en el Modelo de Aceptación de la Tecnología (TAM). Sin embargo, sigue siendo necesario contrastar esta hipótesis, para lo que creemos oportuno la comparación de modelos.

En este sentido, Hair et al. (2004) indican que las comparaciones de modelos pueden llevarse a cabo mediante una estrategia de modelos rivales. Según advierten dichos autores, el investigador se acerca mucho al contraste de "teorías" alternativas, que es un contraste mucho más fuerte que una ligera modificación de una única "teoría". De este modo, podemos construir una serie de modelos rivales en función de teorías alternativas. Estos modelos se pueden basar en las perspectivas psicológica y sociológica. El modelo psicológico está representado por el propio TAM original. El modelo sociológico se puede plantear con las variables sociodemográficas, como la edad y la educación, que pueden afectar directamente a las intenciones de uso de Internet. Pero también es posible establecer un modelo socio-psicológico en el que se incluyan variables sociales y psicológicas, que está más próximo a considerar las variables sociales como antecedentes de las variables psicológicas (Agarwal y Prasad, 1999; Porter y Donthu, 2006; Al-Gahtani, 2008; Tarcan y Varol, 2010). Este tercer tipo de modelo comentado es el que suponemos que puede tener un mayor poder explicativo. Pero esto habría que contrastarlo empíricamente comparando los tres modelos propuestos. 


\section{RefERENCIAS BiBLIOGRÁFICAS}

Agarwal, R. y Prasad, J. 1999. "Are individual differences germane to the acceptance to the information technologies?" Decision Sciences 30(2):361-391.

Agarwal, R. y Prasad, J. 1997. "The role of innovation characteristics and perceived voluntariness in the acceptance of information technologies." Decision Sciences 28(3):557-582.

Al-Gahtani, S. 2001. "The applicability of TAM outside North America: An empirical test in the United Kingdom." Information Resources Management Journal 14(3):37-46.

Al-Gahtani, S. 2008. "Testing for applicability of the TAM model in the Arabic context: Exploring an extended TAM with three moderating factors." Information Resources Management Journal 21(4):1-26.

Davis, F.D., Bagozzi, R.P. y Warshaw, P.R. 1992. "Extrinsic and intrinsic motivation to use computers in the workplace." Journal of Applied Social Psychology 22(14):1111-1132.

Hair, J.F.Jr., Anderson, R.E., Tatham, R.L. y Black, W.C. 2004. Análisis multivariante. Madrid: Prentice Hall.

Legris, P., Ingham, J. y Collerette, P. 2003. "Why do people use information technology? A critical review of the technology acceptance model." Information \& Management 40(3):191-204.

Leo, T.S.H., Lim, V.K.G., y Lai, R.Y.C. 1999. "Intrinsic and extrinsic motivation in Internet usage." Omega 27:25-37.

López Bonilla, L. M. y López Bonilla, J. M. 2006. "Estudio Comparado de las Estimaciones de dos versiones del Modelo de Aceptación de la Tecnología (TAM) mediante los programas Amos y PLS." Investigaciones Europeas de Dirección y Economía de la Empresa 12(3):95-110.

Porter, C.E. y Donthu, E. 2006. "Using the technology acceptance model to explain how attitudes determine Internet usage: The role of perceived access barriers and demographics." Journal of Business Research 59:999-1007.

Roberts, P. y Henderson, R. 2000. "Information technology acceptance in a sample of government employees: a test of the technology acceptance model." Interacting with Computers 12:427-443.

Tarcan, E. y Varol, E.S. 2010. "Role of demographic factors in the process of hotel information systems adoption." Tourism 58(2):127-144.

Torres Albero, C., Robles Morales, J.M. y Molina Molina, O. 2011. "¿Por qué usamos las tecnologías de la información y las comunicaciones? Un estudio sobre las bases sociales de la utilidad individual de Internet." Revista Internacional de Sociología 69(2):371-392.

Venkatesh, V. y Davis, F. D. 2000. "A theoretical extension of the technology acceptance model: Four longitudinal field studies." Management Science 46:186-204.

Venkatesh, V., Morris, M.G., Davis, G.B. y Davis, F.D. 2003. "User acceptance of information technology: Toward a unified view." MIS Quarterly 27:425-478. 
JESÚS MANUEL LÓPEZ-BONILLA es Doctor en Ciencias Económicas y Empresariales por la Universidad de Sevilla y es Profesor Titular de Universidad, adscrito al área de conocimiento de Comercialización e Investigación de Mercados. Entre sus ámbitos de investigación destacan el comportamiento del consumidor, las innovaciones de servicios, el estudio de género y la actividad turística, temas sobre los que ha publicado numerosos artículos.

LUIS MIGUEL LÓPEZ-BONILLA es Doctor en Ciencias Económicas y Empresariales por la Universidad de Sevilla y es Profesor Titular de Universidad, adscrito al área de conocimiento de Comercialización e Investigación de Mercados. Sus principales áreas de investigación son: adopción de innovaciones, comportamiento del consumidor, autoservicios y turismo, en las que cuenta con gran número de publicaciones. 